\title{
A Discretized Approach to W. T. Gowers' Game by
}

\author{
V. KANELLOPOULOS and K. TYROS \\ Presented by Aleksander PEŁCZYŃSKI
}

Summary. We give an alternative proof of W. T. Gowers' theorem on block bases by reducing it to a discrete analogue on specific countable nets. We also give a Ramsey type result on $k$-tuples of block sequences in a normed linear space with a Schauder basis.

1. Introduction. W. T. Gowers in [11] (see also [10] and [12]) proved a fundamental Ramsey-type theorem for block bases in Banach spaces which led to important discoveries in the geometry of Banach spaces. By now there are several approaches to Gowers' theorem (see [1, 2, 3, 4, 14, 21]; also in [7, 15, 18] there are direct proofs of Gowers' dichotomy, and in [6, 8, 19, 22, 24] extensions and further applications).

Our aim in this note is to prove a discrete analogue of Gowers' theorem which is free of approximations. To state our results we will need the following notation. Let $\mathfrak{X}$ be a real linear space with an infinite countable Hamel basis $\left(e_{n}\right)_{n}$ (actually the field over which the linear space $\mathfrak{X}$ is defined plays no role in the arguments; it is only for the sake of convenience that we will assume that $\mathfrak{X}$ is a real linear space). For a subset $A \subseteq \mathfrak{X}$ we denote by $\langle A\rangle$ the linear span of $A$. Let $\mathfrak{D}$ be a subset of $\mathfrak{X}$. We denote by $\mathcal{B}_{\mathfrak{D}}^{\infty}$ the set of all block sequences $\left(x_{n}\right)_{n}$ with $x_{n} \in \mathfrak{D}$ for all $n$. For a block sequence $Z \in \mathcal{B}_{\mathfrak{D}}^{\infty}$ let $\mathcal{B}_{\mathfrak{D}}^{\infty}(Z)$ be the set of all block sequences of $\mathcal{B}_{\mathfrak{D}}^{\infty}$ which are block subsequences of $Z$.

Assume that $\mathcal{B}_{\mathfrak{D}}^{\infty}$ is non-empty and let $Z \in \mathcal{B}_{\mathfrak{D}}^{\infty}$ and $\mathcal{G} \subseteq \mathcal{B}_{\mathfrak{D}}^{\infty}$. We define the $\mathfrak{D}$-Gowers' game in $Z$, denoted by $G_{\mathfrak{D}}(Z)$, as follows. Player I starts the game by choosing $W_{0} \in \mathcal{B}_{\mathfrak{D}}^{\infty}(Z)$ and player II responds with a vector $w_{0} \in\left\langle W_{0}\right\rangle \cap \mathfrak{D}$. Then player I chooses $W_{1} \in \mathcal{B}_{\mathfrak{D}}^{\infty}(Z)$ and player II chooses 
a vector $w_{1} \in\left\langle W_{1}\right\rangle \cap \mathfrak{D}$ and so on. Player II wins the game if the sequence $\left(w_{0}, w_{1}, \ldots\right)$ belongs to $\mathcal{G}$.

Suppose that $\mathfrak{D}$ is a subset of $\mathfrak{X}$ with the following properties:

(D1) (Asymptotic property) $\mathfrak{D} \cap\left\langle\left(e_{i}\right)_{i \geq n}\right\rangle \neq \emptyset$ for all $n \in \mathbb{N}$.

(D2) (Finitization property) $\mathfrak{D} \cap\left\langle\left(e_{i}\right)_{i<n}\right\rangle$ is finite for all $n \in \mathbb{N}$.

Property $(\mathfrak{D} 1)$ simply means that the set $\mathcal{B}_{\mathfrak{D}}^{\infty}$ of all block sequences is nonempty. Property $(\mathfrak{D} 2)$ implies that $\mathfrak{D}$ is countable. Hence, if we endow $\mathfrak{D}$ with the discrete topology, the space $\mathfrak{D}^{\mathbb{N}}$ of all infinite countable sequences in $\mathfrak{D}$ equipped with the product topology is a Polish space.

We can now state our first main result.

TheOREM 1. Let $\mathfrak{X}$ be a real linear space with a countable Hamel basis $\left(e_{n}\right)_{n}$ and let $\mathfrak{D} \subseteq \mathfrak{X}$ have properties $(\mathfrak{D} 1)$ and $(\mathfrak{D} 2)$. Also let $\mathcal{G} \subseteq \mathcal{B}_{\mathfrak{D}}^{\infty}$ be an analytic subset of $\mathfrak{D}^{\mathbb{N}}$. Then for every $U \in \mathcal{B}_{\mathfrak{D}}^{\infty}$ there exists $Z \in \overline{\mathcal{B}}_{\mathfrak{D}}^{\infty}(U)$ such that either $\mathcal{B}_{\mathfrak{D}}^{\infty}(Z) \cap \mathcal{G}=\emptyset$, or player II has a winning strategy in $G_{\mathfrak{D}}(Z)$ for $\mathcal{G}$.

While discrete in nature, Theorem 1 can be used to derive Gowers' original result provided that $\mathfrak{D}$ has an additional property (see Section 4 ).

Our second main result concerns $k$-tuples of block sequences in normed linear spaces with a Schauder basis. Precisely, let $\mathfrak{X}$ be a real normed linear space with a Schauder basis $\left(e_{n}\right)_{n}$. We denote by $\mathcal{B}_{\mathfrak{X}}^{\infty}$ the set of block sequences in $\mathfrak{X}$ and by $\mathcal{B}_{B_{\mathfrak{X}}}^{\infty}$ the set of all block sequences in the unit ball $B_{\mathfrak{X}}$ of $\mathfrak{X}$. Two block sequences $Z_{1}=\left(z_{n}^{1}\right)_{n}$ and $Z_{2}=\left(z_{n}^{2}\right)_{n}$ in $\mathcal{B}_{\mathfrak{X}}^{\infty}$ are said to be disjointly supported if $\operatorname{supp} z_{n}^{1} \cap \operatorname{supp} z_{m}^{2}=\emptyset$ for all $m, n$. For a positive integer $k \geq 2$ and $Z \in \mathcal{B}_{\mathfrak{X}}^{\infty}$, the set of all $k$-tuples of pairwise disjointly supported block subsequences of $Z$ in $B_{\mathfrak{X}}$ will be denoted by $\left(\mathcal{B}_{B_{\mathfrak{X}}}^{\infty}(Z)\right)_{\perp}^{k}$. Also, for a family $\mathfrak{F} \subseteq\left(\mathcal{B}_{\mathfrak{X}}^{\infty}\right)^{k}$ of $k$-tuples of block sequences in $\mathfrak{X}$, the upward closure of $\mathfrak{F}$ is defined to be the set

$$
\begin{aligned}
\mathfrak{F}^{\uparrow}=\left\{\left(U_{i}\right)_{i=0}^{k-1} \in\left(\mathcal{B}_{\mathfrak{X}}^{\infty}\right)^{k}:\right. & \exists\left(V_{i}\right)_{i=0}^{k-1} \in \mathfrak{F} \text { such that } \\
& \left.\forall i V_{i} \text { is a block subsequence of } U_{i}\right\} .
\end{aligned}
$$

If $\Delta=\left(\delta_{n}\right)_{n}$ is a sequence of positive reals, then the $\Delta$-expansion of $\mathfrak{F}$ is defined to be the set

$$
\mathfrak{F}_{\Delta}=\left\{\left(U_{i}\right)_{i=0}^{k-1} \in\left(\mathcal{B}_{\mathfrak{X}}^{\infty}\right)^{k}: \exists\left(V_{i}\right)_{i=0}^{k-1} \in \mathfrak{F} \text { such that } \forall i \operatorname{dist}\left(U_{i}, V_{i}\right) \leq \Delta\right\} .
$$

We prove the following.

THEOREM 2. Let $\mathfrak{X}$ be a real normed linear space with a Schauder basis, $k \geq 2$, and $\mathfrak{F}$ an analytic subset of $\left(\mathcal{B}_{B_{\mathfrak{X}}}^{\infty}\right)^{k}$. Then for every sequence $\Delta=$ $\left(\delta_{n}\right)_{n}$ of positive real numbers there is $Y \in \mathcal{B}_{\mathfrak{X}}^{\infty}$ such that either $\left(\mathcal{B}_{B_{\mathfrak{X}}}^{\infty}(Y)\right)_{\perp}^{k} \cap \mathfrak{F}$ $=\emptyset$, or $\left(\mathcal{B}_{B_{\mathfrak{X}}}^{\infty}(Y)\right)^{k} \subseteq\left(\mathfrak{F}_{\Delta}\right)^{\uparrow}$. 
In the above theorem the topology of $\mathcal{B}_{B_{\mathfrak{X}}}^{\infty}$ is the one induced by the product of the norm topology. Theorem 2 applied for $k=2$ and the family

$$
\mathfrak{F}=\left\{\left(U_{1}, U_{2}\right) \in\left(\mathcal{B}_{B_{\mathfrak{X}}}^{\infty}\right)^{2}: U_{1}, U_{2} \text { are } C \text {-equivalent }\right\},
$$

where $C \geq 1$ is a constant, yields Gowers' second dichotomy (see Lemma 7.3 in [11]).

2. Notation. Let $\mathfrak{X}$ be a real linear space with an infinite countable Hamel basis $\left(e_{n}\right)_{n}$. For two non-zero vectors $x, y$ in $\mathfrak{X}$, we write $x<y$ if $\max \operatorname{supp} x<\min \operatorname{supp} y($ where $\operatorname{supp} x$ is the support of $x$, i.e. if $x=$ $\sum_{n} \lambda_{n} e_{n}$ then $\left.\operatorname{supp} x=\left\{n \in \mathbb{N}: \lambda_{n} \neq 0\right\}\right)$. A sequence $\left(x_{n}\right)_{n}$ of vectors in $\mathfrak{X}$ is called a block sequence (or block basis) if $x_{n}<x_{n+1}$ for all $n$.

Capital letters (such as $U, V, Y, Z, \ldots$ ) refer to infinite block sequences, and overlined lower case letters ( $\operatorname{such}$ as $\bar{u}, \bar{v}, \bar{y}, \bar{z}, \ldots$ ) to finite block sequences. We write $Y \preceq Z$ to denote that $Y$ is a block subsequence of $Z$, that is, $Y=\left(y_{n}\right)_{n}, Z=\left(z_{n}\right)_{n}$ are block sequences and $y_{n} \in\left\langle\left(z_{i}\right)_{i}\right\rangle$ for all $n$. The notations $\bar{y} \preceq Z$ and $\bar{y} \preceq \bar{z}$ are defined analogously. For $\bar{x}=\left(x_{n}\right)_{n=0}^{k}$ and $Y=\left(y_{n}\right)_{n}$ we write $\bar{x}<Y$ if $x_{k}<y_{0}$. For $\bar{x}<Y, \bar{x}^{\wedge} Y$ denotes the block sequence $\left(z_{n}\right)_{n}$ that starts with the elements of $\bar{x}$ and continues with those of $Y$. Also for $\bar{x}<\bar{y}$, the finite block sequence $\bar{x}^{\wedge} \bar{y}$ is similarly defined. For a block sequence $Z=\left(z_{n}\right)_{n}$ and an infinite subset $L$ of $\mathbb{N}$ we set $\left.Z\right|_{L}=\left(z_{n}\right)_{n \in L}$. Also $\left.Z\right|_{k}=\left(z_{n}\right)_{n=0}^{k-1}$ for $k \in \mathbb{N}\left(\left.Z\right|_{0}=\emptyset\right.$ for $\left.k=0\right)$.

Let $\mathfrak{D} \subseteq \mathfrak{X}$. We denote by $\mathcal{B}_{\mathfrak{D}}^{\infty}\left(\operatorname{resp} . \mathcal{B}_{\mathfrak{D}}^{<\infty}\right)$ the set of all infinite (resp. finite) block sequences $\left(x_{n}\right)_{n}$ with $x_{n} \in \mathfrak{D}$ for all $n$. The set of all infinite (resp. finite) block sequences in $\mathfrak{X}$ is denoted by $\mathcal{B}_{\mathfrak{X}}^{\infty}\left(\right.$ resp. $\left.\mathcal{B}_{\mathfrak{X}}^{<\infty}\right)$. For $Z \in \mathcal{B}_{\mathfrak{X}}^{\infty}$ we set $\mathcal{B}_{\mathfrak{D}}^{\infty}(Z)=\left\{Y \in \mathcal{B}_{\mathfrak{D}}^{\infty}: Y \preceq Z\right\}$ and $\mathcal{B}_{\mathfrak{D}}^{<\infty}(Z)=\left\{\bar{y} \in \mathcal{B}_{\mathfrak{D}}^{<\infty}: \bar{y} \preceq Z\right\}$. Similarly $\mathcal{B}_{\mathfrak{D}}^{<\infty}(\bar{z})=\left\{\bar{y} \in \mathcal{B}_{\mathfrak{D}}^{<\infty}: \bar{y} \preceq \bar{z}\right\}$ for $\bar{z} \in \mathcal{B}_{\mathfrak{X}}^{<\infty}$. For a block sequence $Z \in \mathcal{B}_{\mathfrak{D}}^{\infty}$, we set $\langle Z\rangle_{\mathfrak{D}}=\langle Z\rangle \cap \mathfrak{D}$ where $\langle Z\rangle$ is the linear span of $Z$.

3. Discretization of Gowers' game. Throughout this section, $\mathfrak{X}$ is a real linear space with countable Hamel basis $\left(e_{n}\right)_{n}$ and $\mathfrak{D}$ is a subset of $\mathfrak{X}$ with properties $(\mathfrak{D} 1)$ and $(\mathfrak{D} 2)$ as stated in the Introduction. Notice that $(\mathfrak{D} 2)$ also gives that for every $U=\left(u_{i}\right)_{i} \in \mathcal{B}_{\mathfrak{D}}^{\infty}$ and $n \in \mathbb{N}$, the set $\mathcal{B}_{\mathfrak{D}}^{<\infty}\left(\left(u_{i}\right)_{i<n}\right)$ is finite.

3.1. Admissible families of $\mathfrak{D}$-pairs. The aim of this subsection is to review some methods of handling diagonalizations (see also [11, [20]). A $\mathfrak{D}$-pair is a pair $(\bar{x}, Y)$ where $\bar{x} \in \mathcal{B}_{\mathfrak{D}}^{<\infty}$ and $Y \in \mathcal{B}_{\mathfrak{D}}^{\infty}$. For $U \in \mathcal{B}_{\mathfrak{D}}^{\infty}$, a family $\mathcal{P} \subseteq \mathcal{B}_{\mathfrak{D}}^{<\infty}(U) \times \mathcal{B}_{\mathfrak{D}}^{\infty}(U)$ is called an admissible family of $\mathfrak{D}$-pairs in $U$ if it has the following properties:

(P1) (Heredity) If $(\bar{x}, Y) \in \mathcal{P}$ and $Z \in \mathcal{B}_{\mathfrak{D}}^{\infty}(Y)$ then $(\bar{x}, Z) \in \mathcal{P}$. 
$(\mathcal{P} 2)$ (Cofinality) For every $(\bar{x}, Y) \in \mathcal{B}_{\mathfrak{D}}^{<\infty}(U) \times \mathcal{B}_{\mathfrak{D}}^{\infty}(U)$, there is $Z \in$ $\mathcal{B}_{\mathfrak{D}}^{\infty}(Y)$ such that $(\bar{x}, Z) \in \mathcal{P}$.

For simplicity, when we write "pair" we will always mean a $\mathfrak{D}$-pair. It will often happen that an admissible family of pairs has one more property:

$(\mathcal{P} 3)$ If $(\bar{x}, Y) \in \mathcal{P}, \bar{x}<Y$ and $k=\min \left\{m: \bar{x} \in \mathcal{B}_{\mathfrak{D}}^{<\infty}\left(\left(u_{i}\right)_{i=1}^{m}\right)\right\}$ then $\left(\bar{x}, \bar{y}^{\urcorner} Y\right) \in \mathcal{P}$ for every $\bar{y} \in \mathcal{B}_{\mathfrak{D}}^{<\infty}\left(\left(u_{i}\right)_{i=1}^{k}\right)$.

The next lemma follows by a standard diagonalization argument.

Lemma 3. Let $U \in \mathcal{B}_{\mathfrak{D}}^{\infty}$ and let $\mathcal{P}$ be an admissible family of pairs in $U$. Then there is $W \in \mathcal{B}_{\mathfrak{D}}^{\infty}(U)$ such that $(\bar{w}, Y) \in \mathcal{P}$ for all $\bar{w} \in \mathcal{B}_{\mathfrak{D}}^{<\infty}(W)$ and all $Y \in \mathcal{B}_{\mathfrak{D}}^{\infty}(W)$ with $\bar{w}<Y$. If in addition $\mathcal{P}$ satisfies $(\mathcal{P} 3)$ then $(\bar{w}, W) \in \mathcal{P}$ for all $\bar{w} \in \mathcal{B}_{\mathfrak{D}}^{<\infty}(W)$.

3.2. The discrete Gowers' game. Given $Y \in \mathcal{B}_{\mathfrak{D}}^{\infty}$ and a family of infinite block sequences $\mathcal{G} \subseteq \mathcal{B}_{\mathfrak{D}}^{\infty}$, we define the $\mathfrak{D}$-Gowers' game, $G_{\mathfrak{D}}(Y)$, as follows. Player I starts the game by choosing $Z_{0} \in \mathcal{B}_{\mathfrak{D}}^{\infty}(Y)$ and player II responds with a vector $z_{0} \in\left\langle Z_{0}\right\rangle_{\mathfrak{D}}$. Then player I chooses $Z_{1} \in \mathcal{B}_{\mathfrak{D}}^{\infty}(Y)$ and player II chooses a vector $z_{1} \in\left\langle Z_{1}\right\rangle_{\mathfrak{D}}$ with $z_{0}<z_{1}$, and so on. More generally, for a finite block sequence $\bar{x} \in \mathcal{B}_{\mathfrak{D}}^{<\infty}$ and $Y \in \mathcal{B}_{\mathfrak{D}}^{\infty}$ the game $G_{\mathfrak{D}}(\bar{x}, Y)$ is defined as above with the additional condition that player II in the first move chooses $z_{0}>\bar{x}$. Clearly $G_{\mathfrak{D}}(\emptyset, Y)$ is identical to $G_{\mathfrak{D}}(Y)$. We will say that player II wins the game $G_{\mathfrak{D}}(\bar{x}, Y)$ for $\mathcal{G}$ if the block sequence $\bar{x}^{\frown}\left(z_{0}, z_{1}, \ldots\right)$ belongs to $\mathcal{G}$.

We will basically follow the classical Galvin-Prikry terminology (cf. [9], [5]) in the context of Gowers' game. More precisely, for $\bar{x} \in \mathcal{B}_{\mathfrak{D}}^{<\infty}, Y \in \mathcal{B}_{\mathfrak{D}}^{\infty}$ and $\mathcal{G} \subset \mathcal{B}_{\mathfrak{D}}^{\infty}$ we say that $Y \mathcal{G}$-accepts $\bar{x}$ if player II has a winning strategy in $G_{\mathfrak{D}}(\bar{x}, Y)$ for $\mathcal{G}$, while $Y \mathcal{G}$-rejects $\bar{x}$ if no $Z \in \mathcal{B}_{\mathfrak{D}}^{\infty}(Y)$ which $\mathcal{G}$-accepts $\bar{x}$. We also say that $Y \mathcal{G}$-decides $\bar{x}$ if it either $\mathcal{G}$-accepts or $\mathcal{G}$-rejects it.

Notice that if $\bar{x}=\emptyset$ then to say that " $Y \mathcal{G}$-accepts the empty sequence" means that player II has a winning strategy in $G_{\mathfrak{D}}(Y)$ for $\mathcal{G}$. Similarly the statement that " $Y \mathcal{G}$-rejects the empty sequence" is equivalent to saying that for no $Z \in \mathcal{B}_{\mathfrak{D}}^{\infty}(Y)$ does player II have a winning strategy in $G_{\mathfrak{D}}(Z)$ for $\mathcal{G}$. The following lemma is easily verified.

Lemma 4. For every $U \in \mathcal{B}_{\mathfrak{D}}^{\infty}$ and every $\mathcal{G} \subseteq \mathcal{B}_{\mathfrak{D}}^{\infty}$, the family

$$
\mathcal{P}=\left\{(\bar{x}, Y) \in \mathcal{B}_{\mathfrak{D}}^{<\infty}(U) \times \mathcal{B}_{\mathfrak{D}}^{\infty}(U): Y \text { G-decides } \bar{x}\right\}
$$

is an admissible family of pairs in $U$ with property $(\mathcal{P} 3)$.

Actually the family $\mathcal{P}$ of the above lemma satisfies the following condition stronger than $(\mathcal{P} 3)$ : If $(\bar{x}, Y) \in \mathcal{P}$ and $Z \in \mathcal{B}_{\mathfrak{D}}^{\infty}$ are such that $\left.Z\right|_{[n, \infty)} \preceq Y$ for some $n \in \mathbb{N}$, then $(\bar{x}, Z) \in \mathcal{P}$. 
For the sake of simplicity, in the following we will omit the letter $\mathcal{G}$ in front of "accepts", "rejects" and "decides". The next lemma is a consequence of Lemmas 4 and 3 .

Lemma 5. For every $U \in \mathcal{B}_{\mathfrak{D}}^{\infty}$ there is $W \in \mathcal{B}_{\mathfrak{D}}^{\infty}(U)$ such that $W$ decides all $\bar{w} \in \mathcal{B}_{\mathfrak{D}}^{<\infty}(W)$.

The crucial point where the above notions of "accept-reject" essentially differ from the original ones reveals itself in the next lemma. Here the notion of the winning strategy replaces successfully the traditional pigeonhole principle.

Lemma 6. Let $W \in \mathcal{B}_{\mathfrak{D}}^{\infty}$ decide all $\bar{w} \in \mathcal{B}_{\mathfrak{D}}^{<\infty}(W)$ and assume that it rejects some $\bar{w}_{0} \in \mathcal{B}_{\mathfrak{D}}^{\infty}(W)$. Then for every $Y \in \mathcal{B}_{\mathfrak{D}}^{\infty}(W)$ there is $Z \in \mathcal{B}_{\mathfrak{D}}^{\infty}(Y)$ such that $W$ rejects $\bar{w}_{0}^{-} z$ for every $z \in\langle Z\rangle_{\mathfrak{D}}$ with $\bar{w}_{0}<z$.

Proof. If the conclusion is false then there is $Y \in \mathcal{B}_{\mathfrak{D}}^{\infty}(W)$ such that for every $Z \in \mathcal{B}_{\mathfrak{D}}^{\infty}(Y)$ there is $z \in\langle Z\rangle_{\mathfrak{D}}$ with $\bar{w}_{0}<z$ such that $W$ accepts $\bar{w}_{0} z$. It is easy to see that this means that player II has a winning strategy in $G_{\mathfrak{D}}\left(\bar{w}_{0}, Y\right)$ for $\mathcal{G}$, and thus $Y$ accepts $\bar{w}_{0}$. But this is a contradiction since $Y \in \mathcal{B}_{\mathfrak{D}}^{\infty}(W)$ and $W$ rejects $\bar{w}_{0}$.

Lemma 7. For every $U \in \mathcal{B}_{\mathfrak{D}}^{\infty}$ there exists $Z \in \mathcal{B}_{\mathfrak{D}}^{\infty}(U)$ such that either $Z$ rejects all $\bar{z} \in \mathcal{B}_{\mathfrak{D}}^{<\infty}(Z)$, or player II has a winning strategy in $G_{\mathfrak{D}}(Z)$ for $\mathcal{G}$.

Proof. By Lemma 5 there is $W \in \mathcal{B}_{\mathfrak{D}}^{\infty}(U)$ such that $W$ decides all $\bar{w} \in$ $\mathcal{B}_{\mathfrak{D}}^{<\infty}(W)$. If $W$ accepts the empty sequence then we readily have the second alternative of the conclusion for $Z=W$. In the opposite case consider the following family in $\mathcal{B}_{\mathfrak{D}}^{<\infty}(W) \times \mathcal{B}_{\mathfrak{D}}^{\infty}(W)$ :

$$
\begin{aligned}
\mathcal{P}=\{(\bar{x}, Y): \text { either } W \text { accepts } \bar{x}, \\
\left.\quad \text { or } \forall y \in\langle Y\rangle_{\mathfrak{D}} \text { with } \bar{x}<y, W \text { rejects } \bar{x}^{\frown} y\right\} .
\end{aligned}
$$

Using Lemma 6 we easily verify that $\mathcal{P}$ is an admissible family in $W$ which also satisfies $(\mathcal{P} 3)$. Hence by Lemma 3 there is $Z \in \mathcal{B}_{\mathfrak{D}}^{\infty}(W)$ with $(\bar{z}, Z) \in \mathcal{P}$ for every $\bar{z} \in \mathcal{B}_{\mathfrak{D}}^{<\infty}(Z)$. By our assumption $W$ rejects the empty sequence. Since $(\emptyset, Z) \in \mathcal{P}$ we infer that $W$, and so $Z$, rejects all $z \in\langle Z\rangle_{\mathfrak{D}}$. By induction on the length of finite block sequences in $\mathcal{B}_{\mathfrak{D}}^{<\infty}(Z)$, it is easily shown that $Z$ rejects all $\bar{z} \in \mathcal{B}_{\mathfrak{D}}^{<\infty}(Z)$.

We have finally arrived at our first stop which is an analog of the well known result of Nash-Williams ([17]). Consider the set $\mathfrak{D}$ with the discrete topology and $\mathfrak{D}^{\mathbb{N}}$ with the product topology.

Lemma 8. Let $\mathcal{G} \subseteq \mathcal{B}_{\mathfrak{D}}^{\infty}$ be open in $\mathfrak{D}^{\mathbb{N}}$. Then for every $U \in \mathcal{B}_{\mathfrak{D}}^{\infty}$ there exists $Z \in \mathcal{B}_{\mathfrak{D}}^{\infty}(U)$ such that either $\mathcal{B}_{\mathfrak{D}}^{\infty}(Z) \cap \mathcal{G}=\emptyset$, or player II has a winning strategy in $G_{\mathfrak{D}}(Z)$ for $\mathcal{G}$. 
Proof. By Lemma 7 we can find $Z \in \mathcal{B}_{\mathfrak{D}}^{\infty}(U)$ such that either $Z$ rejects all $\bar{z} \in \mathcal{B}_{\mathfrak{D}}^{<\infty}(Z)$, or player II has a winning strategy in $G_{\mathfrak{D}}(Z)$ for $\mathcal{G}$. Hence it suffices to show that the first alternative gives $\mathcal{B}_{\mathfrak{D}}^{\infty}(Z) \cap \mathcal{G}=\emptyset$. Indeed, let $W=\left(w_{n}\right)_{n} \in \mathcal{B}_{\mathfrak{D}}^{\infty}(Z)$. Then for all $k, Z$ rejects $\left.W\right|_{k}=\left(w_{n}\right)_{n<k}$. Therefore there is some $Z_{k} \in \mathcal{B}_{\mathfrak{D}}^{\infty}(Z)$ with $\left.W\right|_{k}<Z_{k}$ such that $\left.W\right|_{k} ^{\curvearrowright} Z_{k} \notin \mathcal{G}$. Since the sequence $\left(\left.W\right|_{k} ^{\wedge} Z_{k}\right)_{k}$ converges in $\mathfrak{D}^{\mathbb{N}}$ to $W$ and the complement of $\mathcal{G}$ is closed, we conclude that $W \notin \mathcal{G}$.

We now pass to the case of an analytic family $\mathcal{G}$. First let us state some basic definitions (cf. [13]). Let $\mathbb{N}<\mathbb{N}$ be the set of all finite sequences in $\mathbb{N}$ and let $\mathcal{N}$ be the Baire space, i.e. the space of all infinite sequences in $\mathbb{N}$ with the topology generated by the sets $\mathcal{N}_{s}=\{\sigma \in \mathcal{N}: \exists n$ with $\sigma \mid n=s\}$, $s \in \mathbb{N}<\mathbb{N}$. A subset of a Polish space $X$ is called analytic if it is the image of a continuous function from $\mathcal{N}$ into $X$.

For the next lemmas we fix the following:

(a) a family $\left(\mathcal{G}^{s}\right)_{s \in \mathbb{N}<\mathbb{N}}$ of subsets of $\mathcal{B}_{\mathfrak{D}}^{\infty}$ such that $\mathcal{G}^{s}=\bigcup_{n} \mathcal{G}^{s^{\wedge} n}$ for all $s$,

(b) a bijection $\varphi: \mathbb{N}^{<\mathbb{N}} \rightarrow \mathbb{N}$ such that $\varphi(\emptyset)=0$ and $\varphi\left(s^{\frown} n\right)>\varphi(s)$ for all $s, n$.

For each $\bar{x}$ in $\mathcal{B}_{\mathfrak{D}}^{<\infty}$ we set $s_{\bar{x}}$ to be the unique element of $\mathbb{N}<\mathbb{N}$ such that $\varphi\left(s_{\bar{x}}\right)$ equals the length of $\bar{x}$. For a $\mathfrak{D}$-pair $(\bar{x}, Y)$ we set

$$
\mathcal{B}_{\mathfrak{D}}^{\infty}(\bar{x}, Y)=\left\{V \in \mathcal{B}_{\mathfrak{D}}^{\infty}: \exists k \text { such that }\left.V\right|_{k}=\bar{x} \text { and }\left.V\right|_{[k, \infty)} \preceq Y\right\} .
$$

Finally, recall the following terminology from [11]. For a family $\mathcal{G} \subseteq \mathcal{B}_{\mathfrak{D}}^{\infty}$ we say that $\mathcal{G}$ is large for $(\bar{x}, Y)$ if $\mathcal{G} \cap \mathcal{B}_{\mathfrak{D}}^{\infty}(\bar{x}, Z) \neq \emptyset$ for all $Z \in \mathcal{B}_{\mathfrak{D}}^{\infty}(Y)$. In the case $\bar{x}=\emptyset$ we simply say that $\mathcal{G}$ is large for $Y$.

Lemma 9. For every $U \in \mathcal{B}_{\mathfrak{D}}^{\infty}$ there is $W \in \mathcal{B}_{\mathfrak{D}}^{\infty}(U)$ such that for every $\bar{w} \in \mathcal{B}_{\mathfrak{D}}^{<\infty}(W)$, either $\mathcal{G}^{s \bar{w}} \cap \mathcal{B}_{\mathfrak{D}}^{\infty}(\bar{w}, W)=\emptyset$, or $\mathcal{G}^{s} \bar{w}$ is large for $(\bar{w}, W)$.

Proof. Let $\mathcal{P}$ be the set of all pairs $(\bar{x}, Y)$ in $\mathcal{B}_{\mathfrak{D}}^{<\infty}(U) \times \mathcal{B}_{\mathfrak{D}}^{\infty}(Y)$ such that either $\mathcal{G}^{s_{\bar{x}}} \cap \mathcal{B}_{\mathfrak{D}}^{\infty}(\bar{x}, Y)=\emptyset$, or $\mathcal{G}^{s_{\bar{x}}}$ is large for $(\bar{x}, Y)$. It is easy to see that $\mathcal{P}$ is admissible and satisfies $(\mathcal{P} 3)$. Hence the conclusion follows by Lemma 3.

Let $W \in \mathcal{B}_{\mathfrak{D}}^{\infty}$ be a block sequence in $\mathfrak{D}$ satisfying the conclusion of Lemma 9. For $\bar{w} \in \mathcal{B}_{\mathfrak{D}}^{<\infty}(W)$, let $\mathcal{F}(\bar{w})$ be the family of all $V=\left(v_{i}\right)_{i} \in$ $\mathcal{B}_{\mathfrak{D}}^{\infty}(W)$ with $\bar{w}<V$ and with the following properties. There exist $m, l \in \mathbb{N}$ with $l \geq 1$ such that

(i) $s_{\bar{w}} m=s_{\bar{x}}$, where $\bar{x}=\bar{w}^{\urcorner}\left(v_{i}\right)_{i=0}^{l-1}$,

(ii) the family $\mathcal{G}^{s_{\bar{w}}} m$ is large for $\left(\bar{w}^{\frown}\left(v_{i}\right)_{i=0}^{l-1}, W\right)$.

Notice that $\mathcal{F}(\bar{w})$ is open in $\mathfrak{D}^{\mathbb{N}}$.

Lemma 10. Let $\bar{w} \in \mathcal{B}_{\mathfrak{D}}^{<\infty}(W)$ and assume that $\mathcal{G}^{s_{\bar{w}}}$ is large for $(\bar{w}, W)$. Then $\mathcal{F}(\bar{w})$ is large for $W$. 
Proof. Let $Z \in \mathcal{B}_{\mathfrak{D}}^{\infty}(W)$. Since $\mathcal{G}^{s_{\bar{w}}}$ is large for $(\bar{w}, W)$ there is $V=\left(v_{i}\right)_{i}$ such that $\bar{w}<V$ and $\bar{w}^{\frown} V \in \mathcal{G}^{s_{\bar{w}}} \cap \mathcal{B}_{\mathfrak{D}}^{\infty}(\bar{w}, Z)=\bigcup_{m} \mathcal{G}^{s_{\bar{w}} m} \cap \mathcal{B}_{\mathfrak{D}}^{\infty}(\bar{w}, Z)$ and so $\bar{w}^{\urcorner} V \in \mathcal{G}^{s_{\bar{w}}} m \cap \mathcal{B}_{\mathfrak{D}}^{\infty}(\bar{w}, Z)$ for some $m \in \mathbb{N}$. Notice that for $l=$ $\varphi\left(s^{\frown} m\right)-\varphi(s)$ we have $s_{\bar{w}}^{\frown} m=s_{\bar{x}}$, where $\bar{x}=\bar{w}^{\frown}\left(v_{i}\right)_{i=0}^{l-1}$, and $\bar{w}^{\frown} V \in$ $\mathcal{G}^{\varsigma_{\bar{w}}} m \cap \mathcal{B}_{\mathfrak{D}}^{\infty}\left(\bar{w}^{\urcorner}\left(v_{i}\right)_{i=0}^{l-1}, Z\right)$. Therefore $\mathcal{G}^{s_{\bar{w}} m} \cap \mathcal{B}_{\mathfrak{D}}^{\infty}\left(\bar{w}^{\frown}\left(v_{i}\right)_{i=0}^{l-1}, W\right) \neq \emptyset$, which (by the properties of $W)$ means that $\mathcal{G}^{s} \frac{\frown}{\bar{w}} m$ is large for $\left(\bar{w}^{\frown}\left(v_{i}\right)_{i=0}^{l-1}, W\right)$. Hence $V \in \mathcal{F}(\bar{w}) \cap \mathcal{B}_{\mathfrak{D}}^{\infty}(Z)$.

Lemma 11. There is $Z \in \mathcal{B}_{\mathfrak{D}}^{\infty}(W)$ such that for every $\bar{z} \in \mathcal{B}_{\mathfrak{D}}^{<\infty}(Z)$, either $\mathcal{G}^{s_{\bar{w}}} \cap \mathcal{B}_{\mathfrak{D}}^{\infty}(\bar{z}, Z)=\emptyset$ or player II has a winning strategy in the game $G_{\mathfrak{D}}(Z)$ for the family $\mathcal{F}(\bar{z})$.

Proof. Let $\mathcal{P}$ be the family of pairs $(\bar{w}, Y) \in \mathcal{B}_{\mathfrak{D}}^{<\infty}(W) \times \mathcal{B}_{\mathfrak{D}}^{\infty}(W)$ such that either $\mathcal{G}^{s \bar{w}} \cap \mathcal{B}_{\mathfrak{D}}^{\infty}(\bar{w}, Y)=\emptyset$, or player II has a winning strategy in $G_{\mathfrak{D}}(Y)$ for $\mathcal{F}(\bar{w})$.

By Lemma 3 it suffices to show that $\mathcal{P}$ is an admissible family of pairs in $W$ which in addition satisfies $(\mathcal{P} 3)$. It is easy to see that only the cofinality property needs some explanation. Let $(\bar{w}, Y) \in \mathcal{B}_{\mathfrak{D}}^{<\infty}(W) \times \mathcal{B}_{\mathfrak{D}}^{\infty}(W)$. Since $\bar{w} \in \mathcal{B}_{\mathfrak{D}}^{<\infty}(W)$, either $\mathcal{G}^{s_{\bar{w}}} \cap \mathcal{B}_{\mathfrak{D}}^{\infty}(\bar{w}, W)=\emptyset$, or $\mathcal{G}^{s_{\bar{w}}}$ is large for $(\bar{w}, W)$. In the first case, $\mathcal{G}^{s \bar{w}} \cap \mathcal{B}_{\mathfrak{D}}^{\infty}(\bar{w}, Y)=\emptyset$ and so $(\bar{w}, Y) \in \mathcal{P}$. In the second case, Lemma 10 implies that $\mathcal{F}(\bar{w})$ is large for $W$. Hence by Lemma 8 , there is $V \in \mathcal{B}_{\mathfrak{D}}^{\infty}(Y)$ such that player II has a winning strategy in $G_{\mathfrak{D}}(V)$ for $\mathcal{F}(\bar{w})$, and so $(\bar{w}, V) \in \mathcal{P}$.

We are now ready for the proof of the main result.

Proof of Theorem 1. Assume that there is no $Z \in \mathcal{B}_{\mathfrak{D}}^{\infty}(U)$ such that $\mathcal{B}_{\mathfrak{D}}^{\infty}(Z) \cap \mathcal{G}=\emptyset$, that is, $\mathcal{G}$ is large for $U$. Let $f: \mathcal{N} \rightarrow \mathfrak{D}^{\mathbb{N}}$ be a continuous map with $f[\mathcal{N}]=\mathcal{G}$, and for $s \in \mathbb{N}^{<\mathbb{N}}$, let $\mathcal{G}^{s}=f\left[\mathcal{N}_{s}\right]$. Then $\mathcal{G}^{\emptyset}=\mathcal{G}$ and $\mathcal{G}^{s}=\bigcup_{n} \mathcal{G}^{s^{\curvearrowright} n}$. Following the process of the above lemmas let $W \in \mathcal{B}_{\mathfrak{D}}^{\infty}(U)$ be as in Lemma 9 and $Z \in \mathcal{B}_{\mathfrak{D}}^{\infty}(W)$ as in Lemma 11 . We claim that player II has a winning strategy in the game $G_{\mathfrak{D}}(Z)$ for $\mathcal{G}$.

Indeed, by our assumption $\mathcal{G}=\mathcal{G}^{\emptyset}$ is large in $\mathcal{B}_{\mathfrak{D}}^{\infty}(Z)=\mathcal{B}_{\mathfrak{D}}^{\infty}(\emptyset, Z)$ and so player II has a winning strategy in $G_{\mathfrak{D}}(Z)$ for $\mathcal{F}(\emptyset)$. This means that player II is able to produce, after a finite number of moves, a finite block sequence $\bar{y}_{0} \in \mathcal{B}_{\mathfrak{D}}^{<\infty}(Z)$ such that there is $m_{0} \in \mathbb{N}$ with $s_{\bar{y}_{0}}=\left(m_{0}\right)$ and $\mathcal{G}^{\left(m_{0}\right)}$ large for $\left(\bar{y}_{0}, W\right)$. By Lemma 11 , player II has a winning strategy in $G_{\mathfrak{D}}(Z)$ for $\mathcal{F}\left(\bar{y}_{0}\right)$, that is, player II can extend $\bar{y}_{0}$ to a finite block sequence $\bar{y}_{0}^{\curlyvee} \bar{y}_{1} \in \mathcal{B}_{\mathfrak{D}}^{<\infty}(Z)$ such that there is $m_{1} \in \mathbb{N}$ such that $s_{\bar{y}_{0}} \bar{y}_{1}=\left(m_{0}, m_{1}\right)$ and $\mathcal{G}^{\left(m_{0}, m_{1}\right)}$ is large for $\left(\bar{y}_{0}^{\curlyvee} \bar{y}_{1}, W\right)$.

Continuing in this way we conclude that player II has a strategy in the game $G_{\mathfrak{D}}(Z)$ to construct a block sequence $Y=\bar{y}_{0} \bar{y}_{1}^{\Upsilon} \ldots$ such that for some 
$\sigma=\left(m_{i}\right)_{i} \in \mathcal{N}$ and for every $k \in \mathbb{N}, \mathcal{G}^{\sigma \mid k}$ is large for $\left(\left(\bar{y}_{0}^{\frown} \ldots \bar{y}_{k-1}\right), W\right)$. To show that this is actually a winning strategy for $\mathcal{G}$, we have to prove that $Y \in \mathcal{G}$. Fix $k \in \mathbb{N}$. Since $\mathcal{G}^{\sigma \mid k}$ is large for $\left(\left(\bar{y}_{0}^{\frown} \ldots{ }^{\frown} \bar{y}_{k-1}\right), W\right)$, there exists $Y_{k} \in \mathcal{B}_{\mathfrak{D}}^{\infty}(W)$ such that $\left(\bar{y}_{0}^{\frown \ldots} \bar{y}_{k-1}\right)^{\frown} Y_{k} \in \mathcal{G}^{\sigma \mid k}$. Since $\left(\mathcal{G}^{\sigma \mid n}\right)_{n}$ is decreasing, $Y=\lim _{n}\left(\bar{y}_{0}^{\frown} \ldots^{\frown} \bar{y}_{n-1}\right)^{\wedge} Y_{n} \in \overline{\mathcal{G}^{\sigma \mid k}}$ for all $k \in \mathbb{N}$, and thus $Y \in \bigcap_{k} \overline{\mathcal{G}^{\sigma \mid k}}$. By the continuity of $f, \bigcap_{k} \overline{\mathcal{G}^{\sigma \mid k}}=\{f(\sigma)\}$ and therefore $Y=$ $f(\sigma) \in \mathcal{G}$.

4. Passing from the discrete to Gowers' game. In this section we will see how using Theorem 1 one can derive W. T. Gowers' Ramsey theorem (see Theorem 16). Henceforth, $\mathfrak{X}$ will be a normed linear space with a Schauder basis $\left(e_{n}\right)_{n}$.

First let us recall some relevant definitions. Let $\mathcal{B}_{\mathfrak{X}}^{\infty}\left(\right.$ resp. $\left.\mathcal{B}_{B_{\mathfrak{X}}}^{\infty}\right)$ be the set of all block sequences in $\mathfrak{X}$ (resp. in the unit ball $B_{\mathfrak{X}}$ ). Let $U=\left(u_{n}\right)_{n}, V=$ $\left(v_{n}\right)_{n} \in \mathcal{B}_{\mathfrak{X}}^{\infty}$ and $\Delta=\left(\delta_{n}\right)_{n}$ a sequence of positive real numbers. We say that $U, V$ are $\Delta$-near and we write $\operatorname{dist}(U, V) \leq \Delta$ if $\left\|u_{n}-v_{n}\right\| \leq \delta_{n}$ for all $n \in \mathbb{N}$. For a family $\mathcal{F} \subseteq \mathcal{B}_{\mathfrak{X}}^{\infty}$ and a sequence $\Delta=\left(\delta_{n}\right)_{n}$ of positive real numbers the $\Delta$-expansion of $\mathcal{F}$ is the set

$$
\mathcal{F}_{\Delta}=\left\{U \in \mathcal{B}_{\mathfrak{X}}^{\infty}: \exists V \in \mathcal{F} \text { such that } \operatorname{dist}(U, V) \leq \Delta\right\} .
$$

For $Y \in \mathcal{B}_{B_{\mathfrak{X}}}^{\infty}$ and a family $\mathcal{F} \subseteq \mathcal{B}_{B_{\mathfrak{X}}}^{\infty}$ the Gowers' game $G_{\mathfrak{X}}(Y)$ is defined as the $\mathfrak{D}$-Gowers game by replacing $\mathfrak{D}$ and $\mathcal{G} \subseteq \mathcal{B}_{\mathfrak{D}}^{\infty}$ with $B_{\mathfrak{X}}$ and $\mathcal{F} \subseteq \mathcal{B}_{B_{\mathfrak{X}}}^{\infty}$ respectively.

For the next two lemmas we fix the following:

(i) a subset $\mathfrak{D}$ of $\left\langle\left(e_{n}\right)_{n}\right\rangle$ with the asymptotic property $(\mathfrak{D} 1)$,

(ii) a family $\mathcal{F} \subseteq \mathcal{B}_{B_{\mathfrak{x}}}^{\infty}$ of block sequences in $B_{\mathfrak{X}}$,

(iii) a sequence $\Delta=\left(\delta_{n}\right)_{n}$ of positive real numbers.

Lemma 12. Let $\mathcal{G}=\mathcal{F}_{\Delta} \cap \mathcal{B}_{\mathfrak{D}}^{\infty}$ and suppose that $\mathcal{B}_{\mathfrak{D}}^{\infty}(\widetilde{Z}) \cap \mathcal{G}=\emptyset$ for some $\widetilde{Z} \in \mathcal{B}_{\mathfrak{D}}^{\infty}$. Assume that there exists $Z \in \mathcal{B}_{\mathfrak{X}}^{\infty}$ such that

$$
\mathcal{B}_{B_{\mathfrak{X}}}^{\infty}(Z) \subseteq\left(\mathcal{B}_{\mathfrak{D}}^{\infty}(\widetilde{Z})\right)_{\Delta}
$$

(that is, for every block subsequence $U=\left(u_{n}\right)_{n}$ of $Z$ with $\left\|u_{n}\right\| \leq 1$ there is a block subsequence $\widetilde{U}=\left(\widetilde{u}_{n}\right)_{n}$ of $\widetilde{Z}$ with $\widetilde{u}_{n} \in \mathfrak{D}$ such that $\left.\operatorname{dist}(U, \widetilde{U}) \leq \Delta\right)$. Then $\mathcal{B}_{B_{\mathfrak{X}}}^{\infty}(Z) \cap \mathcal{F}=\emptyset$.

Proof. Let $U \in \mathcal{B}_{B_{\mathfrak{X}}}^{\infty}(Z)$. By our assumptions there is $\widetilde{U} \in \mathcal{B}_{\mathfrak{D}}^{\infty}(\widetilde{Z})$ such that $\operatorname{dist}(U, \widetilde{U}) \leq \Delta$ and $\widetilde{U} \notin \mathcal{G}$. Then $U \notin \mathcal{F}$, otherwise $\widetilde{U} \in \mathcal{F}_{\Delta} \cap \mathcal{B}_{\mathfrak{D}}^{\infty}(\widetilde{Z})$ which is a contradiction.

Lemma 13. Let $\delta_{0} \leq 1$ and $\sum_{j=n+1}^{\infty} \delta_{j} \leq \delta_{n}$ for all $n$. Let $\mathcal{G}=\mathcal{F}_{\Delta / 10 C}$ $\cap \mathcal{B}_{\mathfrak{D}}^{\infty}$, where $C$ is the basis constant of $\left(e_{n}\right)_{n}$, and suppose that for some 
$\widetilde{Z} \in \mathcal{B}_{\mathfrak{D}}^{\infty}$ player II has a winning strategy in the discrete game $G_{\mathfrak{D}}(\widetilde{Z})$ for $\mathcal{G}$. Assume that there exists $Z \in \mathcal{B}_{\mathfrak{X}}^{\infty}$ such that

$$
\mathcal{B}_{B_{\mathfrak{X}}}^{\infty}(Z) \subseteq\left(\mathcal{B}_{\mathfrak{D}}^{\infty}(\widetilde{Z})\right)_{\Delta / 10 C} .
$$

Then player II has a winning strategy in Gowers' game $G_{\mathfrak{X}}(Z)$ for $\mathcal{F}_{\Delta}$.

Proof. We will define a winning strategy for player II in Gowers' game $G_{\mathfrak{X}}(Z)$ for $\mathcal{F}_{\Delta}$ provided that he has one in the discrete game $G_{\mathfrak{D}}(Z)$ for $\mathcal{G}$. Suppose that we have just completed the $n$th move of $G_{\mathfrak{X}}(Z)\left(\operatorname{resp} . G_{\mathfrak{D}}(\widetilde{Z})\right)$ and $x_{0}<\cdots<x_{n-1}$ (resp. $\left.\widetilde{x}_{0}<\cdots<\widetilde{x}_{n-1}\right)$ have been chosen by player II in $G_{\mathfrak{X}}(Z)$ (resp. in $G_{\mathfrak{D}}(\widetilde{Z})$ ).

Suppose that in $G_{\mathfrak{X}}(Z)$ player I chooses a block sequence $Z_{n}=\left(z_{k}^{n}\right)_{k} \in$ $\mathcal{B}_{\mathfrak{X}}^{\infty}(Z)$. By normalizing we may suppose that $\left\|z_{k}^{n}\right\|=1$ for every $k$, and so by our assumptions on $\widetilde{Z}$ and $Z$ there exists $\widetilde{Z}_{n}=\left(\widetilde{z}_{k}^{n}\right)_{k} \in \mathcal{B}_{\mathfrak{D}}^{\infty}(\widetilde{Z})$ such that $\operatorname{dist}\left(Z_{n}, \widetilde{Z}_{n}\right) \leq \Delta / 10 C$. Then for all $k,\left\|z_{k}^{n}-\widetilde{z}_{k}^{n}\right\| \leq \delta_{k} / 10 C$ and so $\left\|\widetilde{z}_{k}^{n}\right\| \geq 1-\delta_{k} / 10 C$. Let $k_{0} \geq n$ be such that $x_{n-1}<z_{k_{0}}^{n}$ and let player I play $\left.\widetilde{Z}_{n}\right|_{\left[k_{0}, \infty\right]}=\left(\widetilde{z}_{k}^{n}\right)_{k \geq k_{0}}$ in the $n$th move of the discrete game $G_{\mathfrak{D}}(\widetilde{Z})$. Then player II extends $\left(\widetilde{x}_{0}, \ldots, \widetilde{x}_{n-1}\right)$ according to his strategy in $G_{\mathfrak{D}}(\widetilde{Z})$ for $\mathcal{G}$, by picking $\widetilde{x}_{n} \in\left\langle\left(\widetilde{z}_{k}^{n}\right)_{k \geq k_{0}}\right\rangle_{\mathfrak{D}}$. Then $\widetilde{x}_{n}=\sum_{k \in I_{n}} \lambda_{k}^{n} \widetilde{z}_{k}^{n}$, where $I_{n}$ is a finite segment in $\mathbb{N}$ with min $I_{n} \geq k_{0}$ and $\lambda_{k}^{n} \in \mathbb{R}$. Going back to Gowers' game $G_{\mathfrak{X}}(Z)$, let player II play $x_{n}=\sum_{k \in I_{n}} \lambda_{k}^{n} z_{k}^{n}$. Then $x_{n}>x_{n-1}$ and so player II forms in this way a block sequence in $\mathcal{B}_{\mathfrak{X}}(Z)$.

It remains to show that $\left(x_{n}\right)_{n} \in \mathcal{F}_{\Delta}$. Since $\left(\widetilde{x}_{n}\right)_{n} \in \mathcal{G} \subseteq \mathcal{F}_{\Delta / 10 C} \subseteq$ $\left(\mathcal{B}_{B_{\mathfrak{X}}}^{\infty}\right)_{\Delta / 10 C}$, we see that $\left\|\widetilde{x}_{n}\right\| \leq 1+\delta_{n} / 10 C$ for all $n$. Hence

$$
\left|\lambda_{k}^{n}\right| \leq 2 C \frac{\left\|\widetilde{x}_{n}\right\|}{\left\|\widetilde{z}_{k}^{n}\right\|} \leq 2 C \frac{1+\delta_{n} / 10 C}{1-\delta_{k} / 10 C} \leq 2 C \frac{1+\delta_{0} / 10 C}{1-\delta_{0} / 10 C} \leq 4 C
$$

for all $k \in I_{n}$. Therefore,

$$
\left\|x_{n}-\widetilde{x}_{n}\right\| \leq \sum_{k \in I_{n}}\left|\lambda_{k}^{n}\right|\left\|z_{k}^{n}-\widetilde{z}_{k}^{n}\right\| \leq 4 C \sum_{k \in I_{n}} \frac{\delta_{k}}{10 C} \leq \frac{4}{5} \delta_{\min I_{n}} \leq \frac{4}{5} \delta_{n} .
$$

Since $\left(\widetilde{x}_{n}\right)_{n} \in \mathcal{F}_{\Delta / 10 C}$, the last inequality gives $\left(x_{n}\right)_{n \in \mathbb{N}} \in \mathcal{F}_{4 \Delta / 5+\Delta / 10 C} \subseteq$ $\mathcal{F}_{\Delta}$.

The above lemmas lead us to define the next property for a subset $\mathfrak{D}$ of $\mathfrak{X}$ and a given sequence $\Delta=\left(\delta_{n}\right)_{n}$ of positive real numbers.

(D3) ( $\Delta$-block covering property) For every $\widetilde{Z} \in \mathcal{B}_{\mathfrak{D}}^{\infty}$ there exists $Z \in \mathcal{B}_{\mathfrak{X}}^{\infty}$ such that $\mathcal{B}_{B_{\mathfrak{X}}}^{\infty}(Z) \subseteq\left(\mathcal{B}_{\mathfrak{D}}^{\infty}(\widetilde{Z})\right)_{\Delta}$.

In the next proposition we give an example of a subset $\mathfrak{D}$ of $\mathfrak{X}$ with properties $(\mathfrak{D} 1)-(\mathfrak{D} 3)$. Actually we show that a property much stronger than $(\mathfrak{D} 3)$ can be satisfied. In particular, for every $\widetilde{Z} \in \mathcal{B}_{\mathfrak{D}}^{\infty}$ with $\widetilde{Z}=\left(\widetilde{z}_{n}\right)_{n}$, if we set $Z=\left(z_{n}\right)_{n}$ with $z_{n}=\widetilde{z}_{2 n}+\widetilde{z}_{2 n+1}$ then $\mathcal{B}_{B_{\mathfrak{X}}}^{\infty}(Z) \subseteq\left(\mathcal{B}_{\mathfrak{D}}^{\infty}(\widetilde{Z})\right)_{\Delta}$. 
Proposition 14. For every sequence $\Delta=\left(\delta_{n}\right)_{n}$ of positive real numbers there is $\mathfrak{D} \subseteq B_{\mathfrak{X}} \cap\left\langle\left(e_{n}\right)_{n}\right\rangle$ satisfying $(\mathfrak{D} 1)-(\mathfrak{D} 3)$ and such that $\left(e_{n}\right)_{n} \in \mathcal{B}_{\mathfrak{D}}^{\infty}$.

Proof. Let $\left(k_{n}\right)_{n}$ be a strictly increasing sequence of positive integers such that $2^{-k_{n}+1} \leq \delta_{n}$ for every $n$. For $i, l \in \mathbb{N}, l \geq 1$, let

$$
\Lambda(i, l)=\left\{t \cdot 2^{-l \cdot\left(k_{i}+1\right)}: t \in \mathbb{Z}\right\}
$$

For every finite non-empty segment $I=\left[n_{1}, n_{2}\right]$ of $\mathbb{N}, n_{1} \leq n_{2}$, define $\mathfrak{D}(I)=$ $\mathfrak{D}\left(\left[n_{1}, n_{2}\right]\right)$ to be the set of all $x=\sum_{i=n_{1}}^{n_{2}} \lambda_{i} e_{i}$ with the following properties:

(i) For all $n_{1} \leq i \leq n_{2}, \lambda_{i} \in \Lambda(i, l)$, where $l=n_{2}-n_{1}+1$ is the length of $I$.

(ii) The coefficients $\lambda_{n_{1}}$ and $\lambda_{n_{2}}$ are both non-zero.

(iii) $\|x\| \leq 1$.

Finally, we set

$$
\mathfrak{D}=\bigcup_{n_{1} \leq n_{2}} \mathfrak{D}\left(\left[n_{1}, n_{2}\right]\right) .
$$

It is easy to see that $\mathfrak{D}$ satisfies $(\mathfrak{D} 1)-(\mathfrak{D} 2)$. In particular, $\left(e_{n}\right)_{n} \in \mathcal{B}_{\mathfrak{D}}^{\infty}$. It remains to show that $\mathfrak{D}$ has the $\Delta$-block covering property. Actually, we will prove that $\mathfrak{D}$ has a stronger property; to do this we first state the following.

Claim. Let $\widetilde{Z} \in \mathcal{B}_{\mathfrak{D}}^{\infty}$ and let $w \in\langle\widetilde{Z}\rangle$ be such that $\operatorname{card}\left(\operatorname{supp}_{\widetilde{Z}}(w)\right) \geq 2$ and $\|w\| \leq 1$. Then there is $\widetilde{w} \in\langle\widetilde{Z}\rangle_{\mathfrak{D}}$ such that

(1) $\operatorname{supp}_{\widetilde{Z}}(\widetilde{w})=\operatorname{supp}_{\widetilde{Z}}(w)$.

(2) $\|w-\widetilde{w}\| \leq 2^{-k_{m_{1}}+1}$, where $m_{1}=\min _{\operatorname{supp}_{\widetilde{Z}}}(w)$.

Proof of the claim. Let $\widetilde{Z}=\left(\widetilde{z}_{j}\right)_{j}$ and let $\left(I_{j}\right)_{j}, I_{j}=\left[n_{1}(j), n_{2}(j)\right]$, $n_{1}(j) \leq n_{2}(j)$, be the sequence of successive finite non-empty segments of $\mathbb{N}$ such that $\widetilde{z}_{j} \in \mathfrak{D}\left(I_{j}\right)$. Let $m_{1}<m_{2}$ in $\mathbb{N}$, let $\left(\mu_{j}\right)_{j=m_{1}}^{m_{2}}$ be scalars such that $\mu_{m_{1}}, \mu_{m_{2}}$ are both non-zero and let $w=\sum_{j \in\left[m_{1}, m_{2}\right]} \mu_{j} \widetilde{z}_{j}$ in $B_{\mathfrak{X}}$.

Set

$$
w^{\prime}=\left(1-2^{-k_{m_{1}}}\right) w=\sum_{j \in\left[m_{1}, m_{2}\right]}\left(1-2^{-k_{m_{1}}}\right) \mu_{j} \widetilde{z}_{j} \quad \text { and } \quad \widetilde{w}=\sum_{j \in\left[m_{1}, m_{2}\right]} \tilde{\mu}_{j} \widetilde{z}_{j},
$$

where $\widetilde{\mu}_{j}=s_{j} \cdot 2^{-\left(k_{n_{1}(j)}+1\right)}$ and

$$
s_{j}= \begin{cases}\left\lceil\left(1-2^{-k_{m_{1}}}\right) \mu_{j} 2^{k_{n_{1}(j)}+1}\right\rceil & \text { if } \mu_{j} \geq 0, \\ \left\lfloor\left(1-2^{-k_{m_{1}}}\right) \mu_{j} 2^{k_{n_{1}(j)}+1}\right\rfloor & \text { if } \mu_{j}<0,\end{cases}
$$

i.e. $\widetilde{\mu}_{j}$ are of the form $s_{j} \cdot 2^{-\left(k_{n_{1}(j)+1}\right)}$ with $\left|\widetilde{\mu}_{j}\right| \geq\left|\mu_{j}\left(1-2^{-k_{m_{1}}}\right)\right|$ and $\mid \widetilde{\mu}_{j}-$ $\left(1-2^{-k_{m_{1}}}\right) \mu_{j} \mid<2^{-\left(k_{n_{1}(j)}+1\right)}$.

It is easy to see that $\widetilde{\mu}_{j}=0$ if and only if $\mu_{j}=0$ and $\operatorname{son} \operatorname{supp}_{\widetilde{Z}}(\widetilde{w})=$ $\operatorname{supp}_{\widetilde{Z}}(w)$. Moreover, for all $j,\left|\left(1-2^{-k_{m_{1}}}\right) \mu_{j}-\widetilde{\mu}_{j}\right| \leq 2^{-\left(k_{n_{1}(j)}+1\right)}$ and so 


$$
\begin{aligned}
\left\|w^{\prime}-\widetilde{w}\right\| & \leq \sum_{j \in\left[m_{1}, m_{2}\right]}\left|\left(1-2^{-k_{m_{1}}}\right) \mu_{j}-\widetilde{\mu}_{j}\right|\left\|\widetilde{z}_{j}\right\| \\
& \leq \sum_{j \in\left[m_{1}, m_{2}\right]} 2^{-\left(k_{n_{1}(j)}+1\right)} \leq 2^{-k_{n_{1}\left(m_{1}\right)}},
\end{aligned}
$$

and therefore $\left\|w^{\prime}-\widetilde{w}\right\| \leq 2^{-k_{m_{1}}}$, since $m_{1} \leq n_{1}\left(m_{1}\right)$. As $\left\|w-w^{\prime}\right\| \leq 2^{-k_{m_{1}}}$, we obtain $\|w-\widetilde{w}\| \leq 2^{-k_{m_{1}}+1}$.

It remains to show that $\widetilde{w} \in \mathfrak{D}$. Since for all $j \in\left[m_{1}, m_{2}\right]$ we have $\widetilde{z}_{j} \in$ $\mathfrak{D}\left(I_{j}\right)$, it follows that $\widetilde{z}_{j}=\sum_{i \in I_{j}} t_{i}^{j} 2^{-l_{j}\left(k_{i}+1\right)} e_{i}$, where $l_{j}=n_{2}(j)-n_{1}(j)+1$ is the length of $I_{j}$ and $t_{n_{1}(j)}^{j}, t_{n_{2}(j)}^{j}$ are both non-zero. Therefore setting $I=$ $\left[n_{1}\left(m_{1}\right), n_{2}\left(m_{2}\right)\right]$, we have

$$
\widetilde{w}=\sum_{j \in\left[m_{1}, m_{2}\right]} \widetilde{\mu}_{j} \widetilde{z}_{j}=\sum_{j \in\left[m_{1}, m_{2}\right]} \widetilde{\mu}_{j}\left(\sum_{i \in I_{j}} t_{i}^{j} 2^{-l_{j}\left(k_{i}+1\right)} e_{i}\right)=\sum_{i \in I} \lambda_{i} e_{i}
$$

where $\lambda_{i}=t_{i}^{j} 2^{-l_{j}\left(k_{i}+1\right)} \widetilde{\mu}_{j}$ for all $i \in I_{j}$ and $j \in\left[m_{1}, m_{2}\right]$, and $\lambda_{i}=0$ for all $i \in I \backslash \bigcup_{j \in\left[m_{1}, m_{2}\right]} I_{j}$.

We first show that condition (i) of the definition of $\mathfrak{D}$ is satisfied, that is, $\lambda_{i} \in \Lambda(i, l)$ for all $i \in I$, where $l=n_{2}\left(m_{2}\right)-n_{1}\left(m_{1}\right)+1$ is the length of $I$. Since $0 \in \Lambda(i, l)$, it suffices to check this for each $i \in \bigcup_{j \in\left[m_{1}, m_{2}\right]} I_{j}$. So fix $j \in\left[m_{1}, m_{2}\right]$ and $i \in I_{j}$. Then

$$
\lambda_{i}=t_{i}^{j} 2^{-l_{j}\left(k_{i}+1\right)} \widetilde{\mu}_{j}=t_{i}^{j} 2^{-l_{j}\left(k_{i}+1\right)} s_{j} 2^{-\left(k_{n_{1}(j)}+1\right)}=\tau_{i}^{j} 2^{-l\left(k_{i}+1\right)}
$$

where $\tau_{i}^{j}=t_{i}^{j} s_{j} 2^{\left(l-l_{j}\right)\left(k_{i}+1\right)-\left(k_{n_{1}(j)}+1\right)}$. Since $m_{1}<m_{2}$ we have $l>l_{j}$. Also $n_{1}(j) \leq i$ and hence $\left(l-l_{j}\right)\left(k_{i}+1\right)-\left(k_{n_{1}(j)}+1\right) \geq 0$. Therefore $\tau_{i}^{j} \in \mathbb{Z}$, which gives that $\lambda_{i} \in \Lambda(i, l)$.

Moreover, since $\widetilde{\mu}_{m_{1}}, \widetilde{\mu}_{m_{2}}, t_{n_{1}\left(m_{1}\right)}^{m_{1}}, t_{n_{2}\left(m_{2}\right)}^{m_{2}}$ are all non-zero we deduce that $\lambda_{n_{1}\left(m_{1}\right)}$ and $\lambda_{n_{2}\left(m_{2}\right)}$ are also non-zero and so condition (ii) of the definition of $\mathfrak{D}$ is satisfied. Finally, by $\sqrt{1},\|\widetilde{w}\| \leq\left\|w^{\prime}\right\|+2^{-k_{n_{1}\left(m_{1}\right)}} \leq 1$ and so condition (iii) is fulfilled. We conclude that $\widetilde{w} \in \mathfrak{D}$, and the proof of the claim is complete.

We continue with the proof of the proposition. Let $\widetilde{Z}=\left(\widetilde{z}_{j}\right)_{j}$ in $\mathcal{B}_{\mathfrak{D}}^{\infty}$ and let $Z=\left(z_{j}\right)_{j}$ where $z_{j}=\widetilde{z}_{2 j}+\widetilde{z}_{2 j+1}$ for all $j$. Pick $W=\left(w_{i}\right)_{i}$ in $\mathcal{B}_{B_{\mathfrak{X}}}^{\infty}(Z)$. Then for each $i$ there exist $m_{1}^{i}<m_{2}^{i}$ and scalars $\left(\mu_{j}\right)_{j}$ such that $w_{i}=$ $\sum_{j \in\left[m_{1}^{i}, m_{2}^{i}\right]} \mu_{j} \widetilde{z}_{j} \in B_{\mathfrak{X}}$ and $\mu_{m_{1}^{i}}, \mu_{m_{2}^{i}}$ are both non-zero. By the claim, for each $i$ there exist scalars $\left(\widetilde{\mu}_{j}\right)_{j}$ such that $\widetilde{w}_{i}=\sum_{j \in\left[m_{1}^{i}, m_{2}^{i}\right]} \widetilde{\mu}_{j} \widetilde{z}_{j} \in \mathfrak{D}$ and $\left\|w_{i}-\widetilde{w}_{i}\right\|$ $\leq 2^{-k_{m_{1}^{i}+1}} \leq 2^{-k_{i}+1} \leq \delta_{i}$. We set $\widetilde{W}=\left(\widetilde{w}_{i}\right)_{i}$; then $\widetilde{W} \in \mathcal{B}_{\mathfrak{D}}^{\infty}(\widetilde{Z})$ and $\operatorname{dist}(\widetilde{W}, W) \leq \Delta$. Hence $\mathcal{B}_{B_{\mathfrak{X}}}^{\infty}(Z) \subseteq\left(\mathcal{B}_{\mathfrak{D}}^{\infty}(\widetilde{Z})\right)_{\Delta}$ and the proof is complete.

It is easy to see that

$$
\rho(x, y)=\|x-y\|+|1 /\|x\|-1 /\|y\||, \quad x, y \in \mathfrak{X} \backslash\{0\},
$$


is an equivalent metric on $(\mathfrak{X} \backslash\{0\},\|\cdot\|)$ and that the product topology on $(\mathfrak{X} \backslash\{0\}, \rho)^{\mathbb{N}}$ makes $\mathcal{B}_{\mathfrak{X}}^{\infty}$ a Polish space.

Lemma 15. Let $\mathcal{F}$ be an analytic subset of $\mathcal{B}_{\mathfrak{X}}^{\infty}$ and $\Delta=\left(\delta_{n}\right)_{n}$ be a sequence of positive real numbers. Then

(i) $\mathcal{F}_{\Delta}$ is analytic in $\mathcal{B}_{\mathfrak{X}}^{\infty}$.

(ii) For every countable $\mathfrak{D} \subseteq \mathfrak{X}, \mathcal{F}_{\Delta} \cap \mathcal{B}_{\mathfrak{D}}^{\infty}$ is analytic in $\mathfrak{D}^{\mathbb{N}}$ (where $\mathfrak{D}$ is endowed with the discrete topology).

Proof. (i) It is easy to see that $\mathcal{Q}=\{(U, V): \operatorname{dist}(U, V) \leq \Delta\}$ is closed in $\mathcal{B}_{\mathfrak{X}}^{\infty} \times \mathcal{B}_{\mathfrak{X}}^{\infty}$. Let $\operatorname{proj}_{1}$ (resp. proj ${ }_{2}$ ) be the projection of $\mathcal{B}_{\mathfrak{X}}^{\infty} \times \mathcal{B}_{\mathfrak{X}}^{\infty}$ onto the first (resp. second) coordinate. Then $\mathcal{F}_{\Delta}=\operatorname{proj}_{1}\left[\mathcal{Q} \cap\left(\mathcal{B}_{\mathfrak{X}} \times \mathcal{F}\right)\right]=\operatorname{proj}_{1}[\mathcal{Q} \cap$ $\left.\operatorname{proj}_{2}^{-1}(\mathcal{F})\right]$.

(ii) Let $I: \mathfrak{D}^{\mathbb{N}} \rightarrow \mathfrak{X}^{\mathbb{N}}$ be the identity map. Then $I$ is clearly continuous and $\mathcal{F}_{\Delta} \cap \mathcal{B}_{\mathfrak{D}}^{\infty}=I^{-1}\left(\mathcal{F}_{\Delta}\right)$.

Theorem 16 (W. T. Gowers). Let $\mathfrak{X}$ be a normed linear space with a basis and let $\mathcal{F} \subseteq \mathcal{B}_{B_{\mathfrak{X}}}^{\infty}$ be an analytic family of block sequences in the unit ball $B_{\mathfrak{X}}$ of $\mathfrak{X}$. Then for every $\Delta>0$ there exists a block sequence $Z \in \mathcal{B}_{\mathfrak{X}}^{\infty}$ such that either $\mathcal{B}_{B_{\mathfrak{X}}}^{\infty}(Z) \cap \mathcal{F}=\emptyset$, or player II has a winning strategy in Gowers' game $G_{\mathfrak{X}}(Z)$ for $\mathcal{F}_{\Delta}$.

Proof. Let $\left(e_{n}\right)_{n}$ be a normalized basis for $\mathfrak{X}$ with constant $C$. Let $\Delta^{\prime}=$ $\left(\delta_{n}^{\prime}\right)_{n}$ be a sequence of positive real numbers such that $\delta_{0}^{\prime} \leq 1, \delta_{n}^{\prime} \leq \delta_{n}$, and $\sum_{i>n} \delta_{i}^{\prime} \leq \delta_{n}^{\prime}$. By Proposition 14, there is $\mathfrak{D} \subseteq \mathfrak{X}$ with $\left(e_{n}\right)_{n} \in \mathcal{B}_{\mathfrak{D}}^{\infty}$ satisfying $(\mathfrak{D} 1)-(\mathfrak{D} 3)$ for $\Delta^{\prime} / 10 C$. Let also $\mathcal{G}=\mathcal{F}_{\Delta^{\prime} / 10 C} \cap \mathcal{B}_{\mathfrak{D}}^{\infty}$. By Lemma 15, $\mathcal{G}$ is analytic in $\mathfrak{D}^{\mathbb{N}}$, and applying Theorem 1 , we obtain a block sequence $\widetilde{Z} \in \mathcal{B}_{\mathfrak{D}}^{\infty}$ such that either $\mathcal{B}_{\mathfrak{D}}^{\infty}(\widetilde{Z}) \cap \mathcal{G}=\emptyset$, or player II has a winning strategy in $G_{\mathfrak{D}}(\widetilde{Z})$ for $\mathcal{G}$. Choose $Z \in \mathcal{B}_{\mathfrak{X}}^{\infty}$ such that $\mathcal{B}_{B_{\mathfrak{X}}}^{\infty}(Z) \subseteq\left(\mathcal{B}_{\mathfrak{D}}^{\infty}(\widetilde{Z})\right)_{\Delta^{\prime} / 10 C}$. From Lemmas 12 and 13 , either $\mathcal{B}_{B_{\mathfrak{X}}}^{\infty}(Z) \cap \mathcal{F}=\emptyset$, or player II has a winning strategy in Gowers game $G_{\mathfrak{X}}(Z)$ for $\mathcal{F}_{\Delta^{\prime}}$, and so (as $\Delta^{\prime} \leq \Delta$ ) for $\mathcal{F}_{\Delta}$ as well.

5. A Ramsey consequence on $k$-tuples of block bases. The main goal of this section is to prove Theorem 2 . First we need to do some preliminary work and introduce some notation. Fix a positive integer $k \geq 2$. For each $0 \leq i \leq k-1$ and every infinite subset $L=\left\{l_{0}<l_{1}<\cdots\right\}$ of $\mathbb{N}$ we set $L_{i(\bmod k)}=\left\{l_{k n+i}: n \in \mathbb{N}\right\}$ and we define

$$
\left([L]^{\infty}\right)_{\circ}^{k}=\prod_{i=0}^{k-1}\left[L_{i(\bmod k)}\right]^{\infty}=\left\{\left(L_{i}\right)_{i=0}^{k-1} \in\left([L]^{\infty}\right)^{k}: \forall i L_{i} \subseteq L_{i(\bmod k)}\right\} .
$$

Notice that $\left([L]^{\infty}\right)_{\circ}^{k}$ is not hereditary, that is, generally $\left(\left[L^{\prime}\right]^{\infty}\right)_{\circ}^{k} \nsubseteq\left([L]^{\infty}\right)_{\circ}^{k}$ for $L^{\prime} \subseteq L$. Let also

$$
\left([L]^{\infty}\right)_{\perp}^{k}=\left\{\left(L_{i}\right)_{i=0}^{k-1} \in\left([L]^{\infty}\right)^{k}: \forall i \neq j L_{i} \cap L_{j}=\emptyset\right\} .
$$


We have the following elementary lemma which relates the above types of products.

Lemma 17. Let $N=\{(2 n+1) k: n \in \mathbb{N}\}$. Then

$$
\left([N]^{\infty}\right)_{\perp}^{k} \subseteq \bigcup_{L \in[\mathbb{N}]^{\infty}}\left([L]^{\infty}\right)_{\circ}^{k} .
$$

Proof. Let $\left(M_{i}\right)_{i=0}^{k-1} \in\left([N]^{\infty}\right)_{\perp}^{k}$. Let $M=\bigcup_{i=0}^{k-1} M_{i}$ and for each $m \in M$ define the interval $I_{m}=\left[m-i_{m}, m-i_{m}+k-1\right]$ of $\mathbb{N}$ where $i_{m}$ is the unique natural number $i$ such that $m \in M_{i}$. Notice that the length of each $I_{m}$ is $k$ while the length of an interval with unequal endpoints in $N$ is at least $2 k+1$. Hence $I_{m_{1}} \cap I_{m_{2}}=\emptyset$ for $m_{1} \neq m_{2}$, and $I_{m} \cap N=\{m\}$ for all $m \in M$.

Let $L=\bigcup_{m \in M} I_{m}$. We claim that $\left(M_{i}\right)_{i=0}^{k-1} \in\left([L]^{\infty}\right)_{\circ}^{k}$. Indeed, let $L=$ $\left(l_{n}\right)_{n}$ be the increasing enumeration of $L$. For each $0 \leq i \leq k-1$ and $m \in M$ let $I_{m}(i)=m-i_{m}+i$ be the $i$ th element of $I_{m}$. Since $\left(I_{m}\right)_{m \in M}$ is a sequence of pairwise disjoint intervals of $\mathbb{N}$ of length $k$, we easily see that $L_{i(\bmod k)}=$ $\bigcup_{m \in M} I_{m}(i)$. Fix $0 \leq i \leq k-1$. Then $m \in M_{i}$ if and only if $i_{m}=i$ if and only if $I_{m}(i)=m$. Hence $M_{i}=\bigcup_{m \in M_{i}}\left\{I_{m}(i)\right\} \subseteq \bigcup_{m \in M}\left\{I_{m}(i)\right\}=L_{i(\bmod k)}$.

The above notation is easily extended to block sequences in the unit ball $B_{\mathfrak{X}}$ of a Banach space $\mathfrak{X}$ as follows. For every $Z \in \mathcal{B}_{\mathfrak{X}}^{\infty}$ let

$$
\left(\mathcal{B}_{B_{\mathfrak{X}}}^{\infty}(Z)\right)_{\circ}^{k}=\left\{\left(Z_{i}\right)_{i=0}^{k-1} \in\left(\mathcal{B}_{B_{\mathfrak{X}}}^{\infty}\right)^{k}:\left.\forall i \quad Z_{i} \preceq Z\right|_{\mathbb{N}_{i(\bmod k)}}\right\},
$$

and generally for $L \in[\mathbb{N}]^{\infty}$, set

$$
\left(\mathcal{B}_{B_{\mathfrak{X}}}^{\infty}\left(\left.Z\right|_{L}\right)\right)_{\circ}^{k}=\left\{\left(Z_{i}\right)_{i=0}^{k-1} \in\left(\mathcal{B}_{B_{\mathfrak{X}}}^{\infty}\right)^{k}:\left.\forall i \quad Z_{i} \preceq Z\right|_{L_{i(\bmod k)}}\right\} .
$$

The next lemma is an immediate consequence of Lemma 17.

Lemma 18. Let $Z \in \mathcal{B}_{\mathfrak{X}}^{\infty}$ and $N=\{(2 n+1) k: n \in \mathbb{N}\}$. Then

$$
\left(\mathcal{B}_{B_{\mathfrak{X}}}^{\infty}\left(\left.Z\right|_{N}\right)\right)_{\perp}^{k} \subseteq \bigcup_{L \in[\mathbb{N}]^{\infty}}\left(\mathcal{B}_{B_{\mathfrak{X}}}^{\infty}\left(\left.Z\right|_{L}\right)\right)_{\circ}^{k} .
$$

For a family $\mathfrak{F} \subseteq\left(\mathcal{B}_{B_{\mathfrak{X}}}^{\infty}\right)^{k}$ let

$$
\mathcal{F}^{\mathfrak{F}}=\left\{Z \in \mathcal{B}_{S_{\mathfrak{X}}}^{\infty}: \mathfrak{F} \cap\left(\mathcal{B}_{B_{\mathfrak{X}}}^{\infty}(Z)\right)_{\circ}^{k} \neq \emptyset\right\},
$$

where $S_{\mathfrak{X}}$ is the unit sphere of $\mathfrak{X}$.

LEMmA 19. If $\mathfrak{F}$ is analytic in $\left(\mathcal{B}_{\mathfrak{X}}^{\infty}\right)^{k}$, then $\mathcal{F} \mathfrak{F} \subseteq \mathcal{B}_{S_{\mathfrak{X}}}^{\infty}$ is analytic in $\mathcal{B}_{\mathfrak{X}}^{\infty}$.

Proof. Let $\mathcal{K}=\left\{\left(Z,\left(V_{i}\right)_{i=0}^{k-1}\right) \in \mathcal{B}_{S_{\mathfrak{X}}}^{\infty} \times\left(\mathcal{B}_{B_{\mathfrak{X}}}^{\infty}\right)^{k}:\left(V_{i}\right)_{i=0}^{k-1} \in\left(\mathcal{B}_{B_{\mathfrak{X}}}^{\infty}(Z)\right)_{\circ}^{k}\right\}$. Then $\mathcal{K}$ is a closed subset of $\mathcal{B}_{\mathfrak{X}}^{\infty} \times\left(\mathcal{B}_{\mathfrak{X}}^{\infty}\right)^{k}$ and $\mathcal{F} \mathfrak{F}=\operatorname{proj}_{1}\left[\left(\mathcal{B}_{\mathfrak{X}}^{\infty} \times \mathfrak{F}\right) \cap \mathcal{K}\right]$.

Proof of Theorem 2. Let $\left(e_{n}\right)_{n}$ be a normalized basis of $\mathfrak{X}$ with basis constant $C$. Choose $\Delta^{\prime}=\left(\delta_{n}^{\prime}\right)_{n}$ such that $0<\delta_{n}^{\prime} \leq(4 C)^{-1} \delta_{n}$ and $\sum_{j=n+1}^{\infty} \delta_{j}^{\prime} \leq \delta_{n}^{\prime}$. By Lemma $19, \mathcal{F}^{\mathfrak{F}}$ is an analytic subset of $\mathcal{B}_{B_{\mathfrak{X}}}^{\infty}$, and by Theorem 16 there is a block subsequence $Z=\left(z_{n}\right)_{n}$ such that either 
$\mathcal{B}_{B_{\mathfrak{X}}}^{\infty}(Z) \cap \mathcal{F}^{\mathfrak{F}}=\emptyset$, or player II has a winning strategy in Gowers' game $G_{\mathfrak{X}}(Z)$ for $\left(\mathcal{F F}^{\mathfrak{F}}\right)_{\Delta^{\prime}}$. Let $Y=\left.Z\right|_{N}$, where $N=\{(2 n+1) k: n \in \mathbb{N}\}$. We claim that $Y$ satisfies the conclusion of the theorem.

Indeed, if $\mathcal{B}_{B_{\mathfrak{X}}}^{\infty}(Z) \cap \mathcal{F}^{\mathfrak{F}}=\emptyset$ then $\mathfrak{F} \cap\left(\mathcal{B}_{B_{\mathfrak{X}}}^{\infty}\left(Z^{\prime}\right)\right)_{\circ}^{k}=\emptyset$ for all $Z^{\prime} \in \mathcal{B}_{B_{\mathfrak{X}}}^{\infty}(Z)$. In particular, $\mathfrak{F} \cap\left(\mathcal{B}_{B_{\mathfrak{X}}}^{\infty}\left(\left.Z\right|_{L}\right)\right)_{\circ}^{k}=\emptyset$ for all $L \in[\mathbb{N}]^{\infty}$, which by Lemma 18 gives that $\mathfrak{F} \cap\left(\mathcal{B}_{B_{\mathfrak{X}}}^{\infty}(Y)\right)_{\perp}^{k}=\emptyset$.

So assume that player II has a winning strategy in Gowers' game $G_{\mathfrak{X}}(Z)$ for $\left(\mathcal{F}^{\mathfrak{F}}\right)_{\Delta^{\prime}}$. Since $Y=\left.Z\right|_{N}$ the same holds for the game $G_{\mathfrak{X}}(Y)$. Fix $\left(U_{i}\right)_{i=0}^{k-1} \in$ $\left(\mathcal{B}_{B_{\mathfrak{X}}}^{\infty}(Y)\right)^{k}$. We have to show that there exists $\left(V_{i}\right)_{i=0}^{k-1} \in\left(\mathcal{B}_{\mathfrak{X}}^{\infty}\right)^{k}$ such that $V_{i} \preceq U_{i}$ and $\left(V_{i}\right)_{i=0}^{k-1} \in \mathfrak{F}_{\Delta}$. Consider a run of the game such that in the $n$th move player I plays $U_{i}$, where $n=i(\bmod k)$. Then player II succeeds in constructing a block sequence $V=\left(v_{n}\right)_{n}$ in $(\mathcal{F} \mathfrak{F})_{\Delta^{\prime}}$ such that $v_{n} \in U_{i}$ for all $n=i(\bmod k)$. Choose $W$ in $\mathcal{F} \mathfrak{F}$ with $\operatorname{dist}(V, W) \leq \Delta^{\prime}$ and for each $i,\left.W_{i} \preceq W\right|_{\mathbb{N}_{i(\bmod k)}}$ such that $\left(W_{i}\right)_{i=0}^{k-1} \in\left(\mathcal{B}_{B_{\mathfrak{X}}}^{\infty}(W)\right)_{\circ}^{k} \cap \mathfrak{F}$. Let $W=\left(w_{n}\right)_{n}$ and $W_{i}=\left(w_{n}^{i}\right)_{n}$. Then for each $i=1, \ldots, k$ there is a block sequence $\left(F_{n}^{i}\right)_{n}$ of finite subsets of $\mathbb{N}_{i(\bmod k)}$ and a sequence $\left(\lambda_{j}\right)_{j}$ of scalars such that $w_{n}^{i}=\sum_{j \in F_{n}^{i}} \lambda_{j} w_{j}$ for all $i$ and $n$. We set $v_{n}^{i}=\sum_{j \in F_{n}^{i}} \lambda_{j} v_{j}$ and $V_{i}=\left(v_{n}^{i}\right)_{n}$.

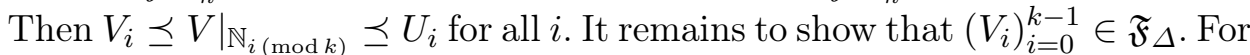
this it suffices to see that $\operatorname{dist}\left(V_{i}, W_{i}\right) \leq \Delta$ for all $i$. Indeed, fix $0 \leq i \leq k-1$ and $n \in \mathbb{N}$. Since $\left\|w_{n}^{i}\right\| \leq 1$ and $\left\|w_{j}\right\|=1$, we get $\left|\lambda_{j}\right| \leq 2 C$ and therefore

$$
\left\|v_{n}^{i}-w_{n}^{i}\right\| \leq \sum_{j \in F_{n}^{i}}\left|\lambda_{j}\right|\left\|v_{j}-w_{j}\right\| \leq 2 C \sum_{j \in F_{n}^{i}} \delta_{j}^{\prime} \leq 4 C \delta_{n}^{\prime} \leq \delta_{n} .
$$

Hence $\left(U_{i}\right)_{i=0}^{k-1} \in\left(\mathfrak{F}_{\Delta}\right)^{\uparrow}$.

6. Comments. 1. C. Rosendal [21] proves a Ramsey dichotomy between winning strategies in Gowers' game and winning strategies in the infinite asymptotic game. By appropriately modifying his argument, one can check that the proof in [21] works in the more general setting of a linear space $\mathfrak{X}$ of countable dimension over the field of reals provided that both games are restricted to a countable subset $\mathfrak{D}$ of $\mathfrak{X}$ with property $(\mathfrak{D} 1)$ stated in the introduction. This modification can be used to derive an alternative proof of Theorem 1 .

2. Theorem 2 is actually an extension of the following fact concerning pairs of infinite subsets of $\mathbb{N}$. Given an analytic family $\mathfrak{F} \subseteq[\mathbb{N}]^{\infty} \times[\mathbb{N}]^{\infty}$ there is an infinite subset $L$ of $\mathbb{N}$ such that either all disjoint pairs of infinite subsets of $L$ belong to the complement of $\mathfrak{F}$, or for every $\left(L_{1}, L_{2}\right) \in[L]^{\infty} \times[L]^{\infty}$, there is $\left(L_{1}^{\prime}, L_{2}^{\prime}\right) \in \mathfrak{F}$ such that $L_{i}^{\prime} \subseteq L_{i}$ for all $i=1,2$. To see this, consider the map $\Phi: M \rightarrow\left(M_{0}, M_{1}\right)$ where if $M=\left\{m_{i}\right\}_{i}$ is the increasing enumeration of $L$ then $M_{0}=\left\{m_{i}\right\}_{i}$ even and $M_{1}=\left\{m_{i}\right\}_{i \text { odd }}$. Then apply Silver's theorem (see 
23]) for the family $\Phi^{-1}\left(\mathfrak{F}^{\uparrow}\right)$ where $\mathfrak{F}^{\uparrow}=\left\{(L, M): \exists\left(L^{\prime}, M^{\prime}\right) \in \mathfrak{F}\right.$ with $L^{\prime} \subseteq L$ and $\left.M^{\prime} \subseteq M\right\}$. It is easy to see that keeping the "half" of the monochromatic set, the result follows. Also, applying K. Milliken's theorem [16], one can derive an analogue of the above result for pairs of block sequences of finite subsets of $\mathbb{N}$.

Acknowledgements. We would like to thank the referee for his (or her) suggestions which simplified the proof of Theorem 1.

This research was supported by PEBE 2007.

\section{References}

[1] G. Androulakis, S. J. Dilworth, and N. J. Kalton, A new approach to the Ramseytype games and the Gowers dichotomy in F-spaces, Combinatorica, to appear.

[2] S. A. Argyros and S. Todorčević, Ramsey Methods in Analysis, Adv. Courses Math., CRM Barcelona, Birkhäuser, Basel, 2005.

[3] J. Bagaria and J. Lopez-Abad, Weakly Ramsey sets in Banach spaces, Adv. Math. 160 (2001), 133-174.

[4] -, -, Determinacy and weakly Ramsey sets in Banach spaces, Trans. Amer. Math. Soc. 354 (2002), 1327-1349.

[5] E. Ellentuck, A new proof that analytic sets are Ramsey, J. Symbolic Logic 39 (1974), 163-165.

[6] V. Ferenczi and C. Rosendal, Banach spaces without minimal subspaces, J. Funct. Anal. 257 (2009), 149-193.

[7] T. Figiel, R. Frankiewicz, R. Komorowski and C. Ryll-Nardzewski, On hereditarily indecomposable Banach spaces, Ann. Pure Appl. Logic 126 (2004), 293-299.

[8] - - - - - , Selecting basic sequences in $\phi$-stable Banach spaces, Studia Math. 159 (2003), 499-515.

[9] F. Galvin and K. Prikry, Borel sets and Ramsey's theorem, J. Symbolic Logic 38 (1973), 193-198.

[10] W. T. Gowers, A new dichotomy for Banach spaces, Geom. Funct. Anal. 6 (1996), 1083-1093.

[11] -, An infinite Ramsey theorem and some Banach-space dichotomies, Ann. of Math. 156 (2002), 797-833.

[12] - Ramsey methods in Banach spaces, in: Handbook of the Geometry of Banach Spaces, Vol. 2, Elsevier, 2003, 1072-1097.

[13] A. S. Kechris, Classical Descriptive Set Theory, Springer, 1995.

[14] J. Lopez-Abad, Coding into Ramsey sets, Math. Ann. 332 (2005), 775-794.

[15] B. Maurey, A note on Gowers' dichotomy theorem, in: Convex Geometric Analysis, Math. Sci. Res. Inst. Publ. 34, Cambridge Univ. Press, Cambridge, 1999, 149-157.

[16] K. Milliken, Ramsey's theorem with sums and unions, J. Combin. Theory Ser. A 18 (1975), 276-290.

[17] C. St. J. A. Nash-Williams, On well-quasi-ordering transfinite sequences, Proc. Cambridge Philos. Soc. 61 (1965), 33-39.

[18] A. M. Pelczar, Some version of Gowers' dichotomy for Banach spaces, Univ. Iagel. Acta Math. 41 (2003), 235-243.

[19] —, Subsymmetric sequences and minimal spaces, Proc. Amer. Math. Soc. 131 (2003), $765-771$. 
[20] P. Pudlák and V. Rödl, Partition theorems for systems of finite subsets of integers, Discrete Math. 39 (1982), 67-73.

[21] C. Rosendal, An exact Ramsey principle for block sequences, Collect. Math. 61 (2010), 25-36.

[22] —, Infinite asymptotic games, Ann. Inst. Fourier (Grenoble) 59 (2009), 1323-1348.

[23] J. Silver, Every analytic set is Ramsey, J. Symbolic Logic 35 (1970), 60-64.

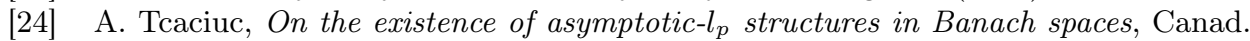
Math. Bull. 50 (2007), 619-631.

V. Kanellopoulos, K. Tyros

Department of Mathematics

Faculty of Applied Sciences

National Technical University of Athens

Zografou Campus

157 80, Athens, Greece

E-mail: bkanel@math.ntua.gr

ktyros@central.ntua.gr

Received October 26, 2009;

received in final form February 5, 2010 